\title{
Simulative Optimierung der Steuerungsparameter eines Materialflusslayouts mit Bandförderern
}

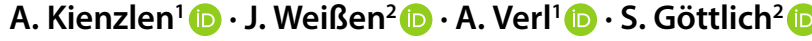 \\ Eingegangen: 2. Juni 2020 / Angenommen: 7. Oktober 2020 / Online publiziert: 27. Oktober 2020 \\ (c) Der/die Autor(en) 2020
}

\section{Zusammenfassung}

Moderne Fertigungssysteme sind komplexe, umfangreiche Systeme. Eine sorgfältige Planung des gesamten Fertigungssystems ist erforderlich, um langfristig effektiv zu produzieren und Gewinne zu erzielen. Bei der Layoutplanung dieser Systeme muss berücksichtigt werden, dass der Materialfluss zwischen einzelnen Stationen zuverlässig und reibungslos funktioniert. Die virtuelle Inbetriebnahme (VIBN) ermöglicht es, das Zusammenspiel von Anlagenlayout und Steuerungssystem, welches das optimale Design der Anlage wesentlich beeinflusst, frühzeitig zu testen. Konzeptionsfehler und Engpässe, die die Performance beeinflussen, können im Voraus erkannt und behoben werden. Für die VIBN wird die reale Anlage durch ein Simulationsmodell ersetzt, um im Zusammenspiel mit dem realen Steuerungssystem Tests durchführen zu können.

In dieser Arbeit wird ein Vorgehensmodell entworfen, anhand dessen die Leistungsfähigkeit von Maschinen und Anlagen mit besonders hohem Materialflussaufkommen analysiert und das Layout bewertet werden kann. Wichtige Leistungskenngrößen wie die Taktzeit und Auslastung einzelner Bestandteile des Fertigungssystem können hinsichtlich ihrer Realisierbarkeit überprüft werden. Durch Testläufe an einem Simulationsmodell des Materialflusses werden Fehler und Engpässe, welche die Performance der Anlage negativ beeinflussen, frühzeitig erkannt. Zur Verbesserung des Layouts wird dabei ein Flussmodell basierend auf einer Erhaltungsgleichung eingesetzt. Das neue Simulationsmodell ermöglicht insbesondere die Simulation großer Mengen an Materialien bei gleichzeitig geringer Berechnungsdauer. Für die Validierung der Ergebnisse werden Parameter festgelegt, die als Indikator für die Leistung von Materialflusssystemen dienen und in einer ingenieurstechnischen Simulation gemessen werden können. Das enge Zusammenspiel verschiedener Simulationen ermöglicht die Validierung und effiziente Verbesserung des Layouts.

Verfügbarkeit von Daten und Material Die Experimentdaten wurden an der Universität Stuttgart im Rahmen des Exzellensclusters für Simulationstechnik (EXC 310/1) mit finanzieller Förderung der DFG erhoben.

Code Verfügbarkeit Simulation des Makro-Modells: benutzerdefinierter Code in Matlab 2019b, Optimierung: Matlab 2019b mit Funktion fmincon, Simulation des Mikro-Modells: benutzerdefinierter Code in AGX Dynamics C++ und Compiler Visual Studio 2015

A. Kienzlen

annika.kienzlen@isw.uni-stuttgart.de
J. Weißen

jennifer.weissen@uni-mannheim.de

1 Institut für Steuerungstechnik der Werkzeugmaschinen und Fertigungseinrichtungen (ISW), Universität Stuttgart, Stuttgart, Deutschland

2 Institut für Mathematik, Universität Mannheim, Mannheim, Deutschland 


\title{
Simulative optimization of the control parameters of a material flow layout with conveyor belts
}

\begin{abstract}
Modern manufacturing systems are complex, extensive systems. Careful planning of the entire manufacturing system is necessary to produce effectively and generate profits in the long term. Effective layout planning has to ensure reliable and smooth material flow between individual stations of the system. Virtual commissioning (VCOM) enables early testing of the interaction between plant layout and control system, which has a significant influence on the optimal design of the plant. Design errors and bottlenecks which affect the performance can be detected and eliminated before the layout is implemented. For the VCOM, the real system is replaced by a simulation model to run tests in interaction with the real control system. In this work, a process model is designed, which can be used to analyze the performance of machines and plants with a particularly high material flow and to evaluate the layout. Important performance parameters such as cycle time and degree of utilization of individual components of the manufacturing system can be identified. Test runs on a simulation model of the material flow allow for early detection of errors and bottlenecks that potentially decrease the performance of the system. To improve the layout, a flow model based on a conservation equation is used, which enables the simulation of large quantities of materials with short calculation time. For the validation of the results, parameters are defined which serve as indicators for the performance of material flow systems and can be measured in an engineering simulation. The close interaction of different simulations enables the validation and efficient improvement of the layout.
\end{abstract}

\section{Einleitung und Problemstellung}

Fertigungssysteme sind komplexe und umfangreiche Systeme, für deren Planung ein sorgfältiges und korrektes Design erforderlich ist [21]. Hohe Qualitätsansprüche zusammen mit der Forderung nach niedrigen Preisen von Konsumentenseite üben einen erheblichen Druck auf Hersteller in allen Phasen des Lebenszyklus einer Anlage aus. Design und Planung, Einbau, Betrieb und mögliche Umgestaltungen, welche Anpassungen an neue Produktionspläne und Neukonzeptionierung der Anlagen umfassen, müssen somit mit geringen Kosten durchgeführt werden [18]. Der Aufbau eines Fertigungssystems ist eine langfristige, unternehmerische Entscheidung und erfordert Investitionen in beträchtlicher Höhe. Die Auslegung und Effizienz des Fertigungssystems ist von höchster Relevanz, um mit den hergestellten Produkten langfristig Gewinne zu erzielen [12].

Bei der virtuellen Inbetriebnahme (VIBN) kann die Validierung eines Fertigungssystems bereits in der Planungsphase der Anlage erfolgen. Die Validierung erfolgt dabei basierend auf einer Simulation, die die reale Anlage abbildet zusammen mit dem realen Steuerungssystem. Somit können die Auslastung der Anlage im Vorhinein überprüft, Engpässe identifiziert, Fehler in der Ablaufplanung erkannt und Fertigungspläne erstellt werden [12]. Die Auslegung von Materialflusssystemen in Fertigungssystemen basiert heutzutage häufig auf Erfahrungswerten und ist daher stark unternehmens- bzw. sogar personengebunden [9]. Ohne VIBN können Tests nur an der realen Anlage durchgeführt werden. Diese Testläufe sind kostenintensiv und zeitaufwendig. Wird den realen Testläufen jedoch eine VIBN vorgeschaltet, können Testläufe und Korrekturen, die an der realen Anlage durchgeführt werden müssen, reduziert werden [12].
Moderne Fertigungssysteme bestehen oftmals aus automatisierten Arbeitsstationen, zwischen denen die Güter mit Materialflusssystemen transportiert werden. Aufgabe der Layoutplanung ist es dabei, einen zuverlässigen Materialfluss zwischen den einzelnen Komponenten des Fertigungssystems sicherzustellen, um den effizienten Ablauf der Fertigung zu gewährleisten [18]. Besonders die Auslastung der Stationen und deren Zusammenspiel, sowie mögliche Engpässe sind in der Planung zu beachten. Im Folgenden wird ein Simulationsansatz vorgestellt, der in der VIBN für Materialflusssysteme genutzt werden kann und mithilfe dessen Anlagenkapazitäten getestet werden. Dabei wird untersucht, ob die geplante Auslastung von Anlagen über die Anpassung der Steuerung und somit der Steuerung des Materialflusses in einem vorgegebenen Layout erreicht werden kann.

Als Materialfluss wird hier die physikalische Bewegung von Stückgütern bezeichnet. Im Unterschied zum Materialfluss in der Logistik werden die Interaktionen zwischen einzelnen, kleineren und mittleren Stückgütern betrachtet, die gemeinsam transportiert werden. Der Transport der Güter erfolgt dabei durch Transportbänder zwischen den einzelnen Arbeitsstationen. Die Verteilung wird mithilfe von Abweisern geregelt. Müssen viele Stückgüter berücksichtigt werden, ist die rein simulative Auslegung der Gesamtanlage erschwert, da eine hohe Anzahl an Stückgütern den Rechenaufwand erheblich erhöht. Sowohl die konstruktive als auch die steuerungstechnische Auslegung eines solchen Materialflusssystems sind aufgrund der vielen Interaktionen und Randbedingungen komplex und bisher mathematisch kaum untersucht.

In dieser Arbeit wird ein Konzept eingesetzt, welches ein Layout hinsichtlich vorgegebener Kriterien für die Stückgüter wie bspw. der Durchflussmenge bewerten kann. Der 
vorgestellte Ansatz nutzt ein optimales Kontrollproblem aus der Mathematik, welches die realen Steuerungsgrößen berücksichtigt. Zur Bestimmung der optimalen Steuerungsgröße wird ein vereinfachtes Flussmodell optimiert, welches den Materialfluss abbildet. Die optimale Auslegung wird an einer Simulation aus der Ingenieurstechnik überprüft. Eine rein simulative Bestimmung der optimalen Steuerungsgröße ist aufgrund der hohen Stückgutanzahlen und den damit verbundenen Rechenzeiten nicht effizient. Die Neuerung des vorgestellten Konzepts besteht darin, die optimale Steuerung mithilfe der mathematischen Optimierung des Materialflussmodells zu gewinnen und somit die Anzahl der rechenintensiven Simulationen auf ein notwendiges Minimum zu reduzieren. Diese Kombination ermöglicht es, effizient Aussagen für Materialflusssysteme zu treffen und mögliche Engpässe bereits in der Planungsphase zu erkennen.

\section{Stand der Technik und eigene Vorarbeiten}

\subsection{Anlagenplanung und -tests}

Aufgrund der Komplexität moderner Anlagen ist der Prozess der Inbetriebnahme umfangreich, da die Entwicklungen verschiedener Disziplinen und deren Zusammenspiel überprüft werden müssen. Es werden verschiedene Tests benötigt, bevor die Produktion anlaufen kann. Je später Fehler entdeckt werden, desto aufwendiger ist deren Behebung und desto mehr verzögert sich die Fertigstellung. Heute kann eine VIBN eingesetzt werden, durch die Tests vorgezogen und Fehler früher entdeckt werden können [20]. Dies gilt insbesondere für die Steuerungstechnik, die ansonsten in der Regel auf die Ergebnisse der Konstruktion (mechanisch, elektrisch, evtl. pneumatisch oder hydraulisch) angewiesen ist. Für die VIBN wird ein Simulationsmodell benötigt, das ein möglichst präzises, virtuelles Abbild des Systems darstellt [27]. Das Simulationsmodell soll sich analog zum realen System verhalten. Es soll die gleichen Ein- und Ausgänge haben und auf die Eingangswerte genauso wie das reale System reagieren. Bei der VIBN wird eine Komponente bspw. die Steuerung getestet, in dem sie mit dem Simulationsmodell gekoppelt wird [25]. Auf diese Weise kann die Steuerung inklusive der Steuerungsprogramme getestet werden, ohne dass die reale Maschine oder Anlage benötigt wird. Die VIBN kann auch genutzt werden, wenn die Anlage an verschiedenen Standorten entwickelt wird. Es ist sinnvoll dieses Simulationsmodell bereits früh zur erstellen, damit es bereits in der Planungsphase genutzt werden kann.

Zur Layoutplanung einer Anlage bzw. eines Materialflusssystems existieren verschiedene Verfahren zur mathematischen Optimierung des Designs bspw. die Tabu-Suche, Simulated Annealing, und genetische Algorithmen [17].
Ein Großteil dieser Methoden berücksichtigt nur die (ereignis-)diskrete Planung [11]. In der ereignisdiskreten Planung wird betrachtet, welche Anzahl an Gütern sich zu diskreten Zeitpunkten an verschiedenen Orten befindet. Diese Untersuchung ist insbesondere für das Supply Chain Management, die Logistik und die Intralogistik interessant. Die Layoutplanung auf einer tieferen Ebene, auf der die exakte, kontinuierliche Bewegung und Interaktion einzelner Stückgüter berücksichtigt werden, wird in der Regel nicht betrachtet. Sie beeinflusst jedoch wesentlich die Erfüllung der Kriterien eines Layouts und wird daher im Folgenden näher untersucht. Ein Layout bezieht sich in diesem Fall auf die konkrete geometrische Form der Transportwege in Kombination mit der Ansteuerung.

\subsection{Materialflussmodelle}

In [23] zeigen die Autoren verschiedene Ansätze zur Modellierung der Bewegung von Stückgütern. Diese lassen sich einteilen in ereignisdiskrete, kinematische und physikbasierte Ansätze und das Flussmodell. Für die Entscheidung für einen Simulationsansatz muss zwischen Genauigkeit und Berechnungsaufwand abgewogen werden [21]. Ereignisdiskrete Ansätze betrachten, im Gegensatz zu den anderen Ansätzen, keinen kontinuierlichen Zeitverlauf. Stattdessen werden verschiedene Größen nach konkreten Arbeitsschritten betrachtet. Es handelt sich in der Regel um Mengen an Gütern, die sich an bestimmten Positionen bspw. in verschiedenen Lagern oder in bestimmten Bearbeitungsschritten in einer Anlage befinden. Da die konkreten Bewegungen der Stückgüter in diesem Ansatz nicht berechnet werden, wird dieser Ansatz im Folgenden nicht weiter betrachtet. Beim kinematischen Modell werden dagegen die Bewegungen der Güter berücksichtigt, wodurch Kollisionen mit der Umgebung aufgezeigt werden können. Allerdings folgen die Stückgüter festgelegten Trajektorien und können nicht miteinander interagieren. Kollidieren zwei Stückgüter so ist dies feststellbar, aber es ergibt sich keine Reaktion auf den weiteren Bewegungsverlauf. Aus diesem Grund wird auch dieses Modell nicht weiter betrachtet.

\subsubsection{Mikroskopisches Modell}

Das physikbasierte Modell beruht auf einer Starrkörpersimulation, bei der die Kräfte und Momente auf die einzelnen Stückgüter berechnet werden. Die physikalischen Eigenschaften und die geometrische Form der Stückgüter werden berücksichtigt [10]. Die Position $x_{i}(t)$ der einzelnen Körper $(i=1, \ldots, \mathrm{N})$ zum Zeitpunkt $t$ wird basierend auf den Newton-Euler-Gleichungen berechnet, wobei die Summe 
der Kräfte $f_{n, i}\left(n=1, \ldots, N_{f}\right)$ auf die Körper proportional zu deren Masse $m_{i}$ und deren Beschleunigung $\ddot{x}_{i}$ ist.

$m_{i} \ddot{x}_{i}(t)=\sum_{n=1}^{N_{f}} f_{n, i}(t), \quad i=1, \ldots, N$

$x_{i}(0)=x_{i, 0}$

Anschließend werden Kollisionen detektiert und aufgelöst. Die Bewegung der Stückgüter resultiert folglich aus den Einwirkungen der Umgebung und der Interaktion der Stückgüter. Durch die Komplexität der Kollisionsberechnungen steigen die Simulationszeiten bei diesem Ansatz mit zunehmender Objektanzahl stark an [10]. Aufgrund der Betrachtung einzelner Stückgüter wird der Ansatz auch als „mikroskopisches Modell““ (Mikro-Modell) bezeichnet.

\subsubsection{Makroskopisches Modell}

Zur Beschreibung des Materialflusses wird ein makroskopisches Modell (Makro-Modell) basierend auf einer Erhaltungsgleichung verwendet (siehe [7, 19, 22]). Aufgrund der Analogie zur Modellierung von Flüssigkeiten, wird es auch als „Flussmodell“ bezeichnet. Während in der mikroskopischen Simulation Stückgüter einzeln betrachtet werden, wird hier nicht mehr die Bewegung des einzelnen Stückgutes betrachtet, sondern die Gesamtheit aller Güter. Daher wird es als „,makroskopisches Modell“ bezeichnet. Die Gesamtheit der Güter wird als Dichteverteilung modelliert. Makroskopische Modelle werden häufig als Grenzwert von mikroskopischen Modellen hergeleitet, wenn die Anzahl der Stückgüter gegen unendlich geht (siehe z. B. [8] für die Herleitung des hier genutzten makroskopischen Modells). Sie eignen sich somit besonders gut für die Beschreibung von Materialfluss mit hoher Stückgutzahl. Die Gesamtheit der Stückgüter wird als Dichteverteilung $\rho=\rho(x, t)$ abhängig von Ort $x$ und Zeit $t$ modelliert, wobei $x \in \Omega \subset R^{2}$ und $t \geq 0$. Das Problem wird auf eine zweidimensionale Betrachtungsweise reduziert.

$\partial_{t} \rho+\nabla \cdot\left(\rho\left(v^{T}(x)+v^{\text {dyn }}(\rho)\right)=0\right.$

$v^{\text {dyn }}(\rho)=H\left(\rho-\rho_{\max }\right) \cdot I(\rho)$

$I(\rho)=-\epsilon \frac{\nabla(\eta * \rho)}{\sqrt{1+|| \nabla(\eta * \rho)||}}$

$\rho(x, 0)=\rho_{0}(x)$

Die Dichte $\rho_{0}(x)$ beschreibt die Verteilung der Güter auf dem Bandförderer zum Zeitpunkt $t=0$. Sie berücksichtigt die initiale Positionierung der Güter auf dem Förderband und kann direkt aus der mikroskopischen Positionierung (2) berechnet werden, siehe [6]. Da es sich um eine explizite Beschreibung handelt, kann für die initiale Dichte auch die Positionierung zu jedem beliebigen Zeitpunkt gewählt werden. Die Geschwindigkeit des Materials berechnet sich aus den beiden Geschwindigkeitsgrößen $v^{T}(x)$ und $v^{\mathrm{dyn}}(\rho)$. Die Geschwindigkeit $v^{T}$ beschreibt externe Komponenten, welche die Geschwindigkeit der Stückgüter ortsabhängig beeinflussen wie bspw. eine Bandgeschwindigkeit. Ein Stückgut, welches sich im Ort $x$ befindet, sollte sich mit der zeitunabhängigen Geschwindigkeit $v^{T}(\mathrm{x})$ bewegen. Ist jedoch die Dichte $\rho(x, t)$ nahe der maximalen Dichte $\rho_{\max }$, ändert sich die Geschwindigkeit des Stückgutes in Abhängigkeit von der Dichteverteilung in der Umgebung. In diesem Fall wird die Heaviside Funktion

$H\left(\rho-\rho_{\max }\right)= \begin{cases}0, & \rho<\rho_{\max } \\ 1, & \rho \geq \rho_{\max } .\end{cases}$

aktiv und schaltet das dynamische Geschwindigkeitsfeld $v^{\mathrm{dyn}}(\rho)$ hinzu, woraus sich Änderungen in der Geschwindigkeit ergeben. Anschaulich gesprochen können in eine Umgebung, die so dicht wie möglich mit Stückgütern versehen ist, keine weiteren Stückgüter hineinlaufen und deshalb wird die Bewegungsrichtung der Stückgüter angepasst. Der *-Operator ist der Faltungsoperator. Der Wert $(\eta * \rho)(x)$ ist derjenige Dichtewert, der sich als gewichteter Mittelwert der Dichteverteilung um den Ort $x$ ergibt. Der Glättungskern $\eta$ gewichtet die Dichteverhältnisse in der Umgebung und im Folgenden wird als Glättungskern der zweidimensionale Gauss Filter

$\eta(x)=\frac{\sigma}{2 \pi} \exp \left(-\frac{1}{2} \sigma\left(x_{1}^{2}+x_{2}^{2}\right)\right)$,

verwendet. Der Parameter $\sigma>0$ skaliert die Breite des Glättungskerns. Je größer $\sigma$, desto kleiner ist die Umgebung, die zur Berechnung der gewichteten Dichte $(\eta * \rho)(x)$ genutzt wird. Im Folgenden wird im Einklang mit der Literatur [22] $\sigma=10000$ genutzt. Somit gehen nur Dichtewerte aus der unmittelbaren Umgebung des Punktes $\mathrm{x}$ in die Berechnung des gewichteten Mittelwertes ein, d.h. es wird lokal geglättet. Die Geschwindigkeit in Abhängigkeit der Dichteverhältnisse wird über den Kollisionsoperator $I(\rho)$ angepasst. Überfüllte Gebiete in der Umgebung können somit in der Berechnung der Bewegungsrichtung berücksichtigt werden. Der Parameter $\epsilon>0$ gewichtet die Abstoßkräfte in Abhängigkeit der Bandgeschwindigkeit. Im Folgenden wird $\epsilon=2 v^{T}$ gewählt (siehe [8]). Der mathematische Vorteil von Flussmodellen gründet sich darin, dass der Aufwand der Auswertung einer Simulation unabhängig von der Anzahl der zu betrachtenden Stückgüter ist, da die Anzahl $N$ der Stückgüter in den Gleichungen nicht zu berücksichtigen ist. Das kommt besonders der rechenintensiven Optimierung bei hoher Stückgutzahl zu Gute, bei denen die mikroskopische Simulation bereits bei einfachen Simulationsläufen sehr rechenintensiv ist. 


\subsection{Stand der Optimierung}

Während im Logistikbereich bereits ereignisdiskrete Simulationen zur Optimierung eingesetzt werden [29], ist die Verwendung von Simulationen für die detaillierte Layoutplanung bisher selten. Die Ursache dafür liegt in der deutlich größeren Komplexität und dementsprechend höheren Rechendauer der benötigten physikbasierten Berechnungen für derartige Simulationen. Dagegen betrachtet das Flussmodell nicht die Bewegung einzelner Stückgüter, sondern die Gesamtheit aller Stückgüter, wodurch die Rechenzeiten bei einer großen Anzahl an Stückgütern geringer sind und somit Potenzial für die Optimierung bieten. Die Kombination eines mathematischen Flussmodells und eines Simulationswerkzeugs für die VIBN stellt einen neuartigen Ansatz dar.

Vorarbeiten zur Optimierung des Materialflusses des makroskopischen Modells finden sich im Bereich „optimal packing “ (vgl. [4]). Hier wird bei gegebener Bandgeschwindigkeit die Anordnung der Stückgüter in einem vorgegebenen Bereich optimiert, sodass diese möglichst schnell den Bereich verlassen. Mathematisch wird das Kontrollproblem über einen ,first-discretize-then-optimize“ Ansatz gelöst [2], indem zunächst die Problemstellung diskretisiert und das diskrete Optimierungsproblem gelöst wird.

\section{Konzept}

\subsection{Zielsetzung}

Ziel der Arbeit ist die Bewertung und Verbesserung von Materialflusslayouts, die für den Transport von vielen Stückgütern verwendet werden. Beispielhafte Anwendungen finden sich etwa in der Flaschenbefüllung. Die Ausgangssituation dieses Konzepts ist eine Anlage mit Materialfluss von Stückgütern derselben Art auf Bandförderern. Die Stationen und deren sequentielle Reihenfolge sind bereits bekannt. Außerdem sind die Kapazitäten und Arbeitszeiten der Stationen gegeben. Ein Entwurf des Anlagenlayouts inklusive der genauen Materialflusswege ist vorhanden. Gesucht ist die optimale Parametrierung der Steuerungssysteme in Bezug auf die Zielgröße wie bspw. der Bandgeschwindigkeit für diese Wege. Die ZielgröBe beschreibt die benötigte oder gewünschte Bewegung der Stückgüter hinsichtlich der gewählten Kriterien. Das kann bspw. eine bestimmte Verteilung der Stückgüter oder eine bestimmte Anzahl an Stückgütern zu einem bestimmten Zeitpunkt sein. Ein iterativer Optimierungsprozess legt schrittweise die Stellgrößen als Eingriffsmöglichkeiten fest.

\subsection{Vorgehensweise}

Wie in Abb. 1 zu sehen, werden zunächst das geplante Materialflusslayout und die Eingangswerte definiert. Anschließend werden die Ziel- und Stellgrößen festgelegt und mit der Makro-Simulation optimiert. Stellgrößen für die Optimierung sollen Parameter sein, die an der Steuerung für das entsprechende Materialflusslayout eingestellt werden können. Um die Ergebnisse zu validieren, werden Bewertungsparameter festgelegt und anhand einer Mikro-Simulation (1)-(2) überprüft. Dabei werden die Stellgrößen, die in der Optimierung bestimmt wurden, an die MikroSimulation übergeben. In der Mikro-Simulation werden die Ausgangswerte für den nächsten Schritt der Optimierung bestimmt und an diese weitergegeben. Außerdem findet anhand der Mikro-Simulation eine Leistungserbringung statt. Diese wird im nächsten Kapitel näher beschrieben. Sollten die Ergebnisse der Leistungserbringung nicht zufriedenstellend sein, so wird der aktuelle Optimierungsschritt mit angepassten Randbedingungen wiederholt. Alternativ besteht die Möglichkeit, Veränderung am Layout vorzunehmen und den Optimierungsvorgang für das veränderte Layout zu wiederholen.

Hier wird ein Szenario der optimalen Kontrolle betrachtet, um die Bandgeschwindigkeit $v^{T}$ so zu steuern, dass sich zu einem vorgegebenen Zeitpunkt $T>0$, eine bestimmte Anzahl von Stückgütern $N_{T}$ in einem vorgegebenen Zielgebiet $\Omega_{Z} \subset \Omega$ befindet. Als Zielfunktion wird im Folgenden beispielhaft die betragsmäßige Abweichung verwendet. Das makroskopische Kontrollproblem lautet:

$$
\begin{aligned}
& \min \left|N_{T}-U\left(v^{T}, T\right)\right| \\
& \text { u.d.N. } U\left(v^{T}, T\right)=\int_{\Omega_{z}} \rho(x, T) d x
\end{aligned}
$$

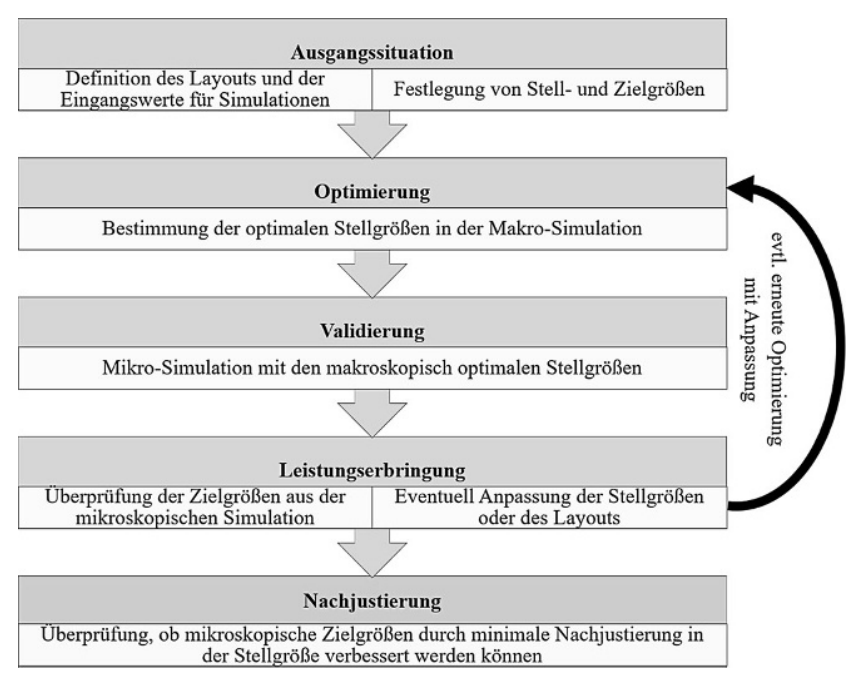

Abb. 1 Vorgehensweise zur Optimierung und Validierung des Materialflusslayouts 
Die Anzahl der Stückgüter in dem vorgegebenen Zielgebiet $\Omega_{z}$ wird mittels Integration der Dichte in dem Gebiet ermittelt. Der Wert $U\left(v^{T}, T\right)$ ergibt sich aus der Integration der Dichte $\rho(x, T)$, welche in der Simulation errechnet wurde. Mehrere dieser Szenarien werden in aufeinander folgenden Optimierungsschritten betrachtet, um ein System aus mehreren Bandförderern zu untersuchen. Das obige, nichtlineare Optimierungsproblem wird mithilfe eines kommerziellen Lösungsalgorithmus gelöst. Der optimale Zielfunktionswert ergibt sich mit der optimalen Geschwindigkeit in der Makro-Simulation. Zur Lösung des Optimierungsproblems wird in dieser Arbeit ein ,first-discretizethen-optimize“ Ansatz verfolgt. Die Lösung des Optimierungsproblems erfordert ausgehend von der Dichteverteilung $\rho(x, 0)$ die Berechnung der Dichteverteilung $\rho(x, T)$, welche durch die Modellgleichungen (3)-(6) gegeben ist. Zur Berechnung dieser Dichteverteilung wird zunächst auf einem Gitter in Zeit und Ort mit Schrittweiten $\Delta x$ und $\Delta t$ diskretisiert und für gegebene Bandgeschwindigkeit $v^{\mathrm{T}}$ anschließend die Dichteverteilung berechnet. Das Gebiet $\Omega$ wird dabei in rechteckige Zellen mit Zellmittelpunkten $x_{i j}=\left(x_{1, i}, x_{2, j}\right), i=1, \ldots N_{x_{1}}, j=1, \ldots N_{x_{2}}$ unterteilt und die Diskretisierung der Zeit ist gegeben durch $t_{k}=k \Delta t, k=$ $1, \ldots N_{t}$. Der Simulationsansatz berechnet die Lösung der Modellgleichungen (3)-(6) als stückweise konstante Funktion

$\rho\left(x, t_{k}\right)=\rho_{i j}^{k} \in \mathbb{R}$,

für $x \in\left(x_{1, i-\frac{1}{2}}, x_{2, j-\frac{1}{2}}\right) \times\left(x_{1, i+\frac{1}{2}}, x_{2, j+\frac{1}{2}}\right)$

Zur Simulation wird in dieser Arbeit ein Finite Volumen Verfahren verwendet, welches gute Lösungen der Modellgleichung liefert [22]. Die Modellierung des statischen Geschwindigkeitsfelds $v^{T}(x)$, auf welches das Verfahren zurückgreift, umfasst die Geometrie des Gebietes $\Omega$. Hiermit können Wände und Hindernisse in die Modellierung eingebunden werden [7]. Hindernisse werden durch Kräfte integriert, die entgegengesetzt der Bewegungsrichtung des Bandes wirken und in Abhängigkeit der Bandgeschwindigkeit skaliert werden. Der Zielfunktionswert der Optimierung wird basierend auf der diskretisierten Lösung (11) berechnet. Die Verbesserung des Zielfunktionswertes und das Finden der optimalen Lösung erfolgt mittels eines Lösungsalgorithmus, der als Eingabe den Simulationsalgorithmus inklusive der Zielfunktion erhält.

\section{Leistungserbringung}

\section{1 Überführung in die Mikro-Simulation}

Um die Ergebnisse der Optimierung zu überprüfen, soll das Szenario in einer Mikro-Simulation betrachtet werden.
Die Mikro-Simulation dient hierbei auch der Validierung der Ergebnisse aus der Optimierung. Da die Makro-Simulation die 3. Ortsdimension vernachlässigt (bspw. Kippen oder Stapelung) und keine Informationen über die einzelnen Stückgüter enthält, werden die Optimierungsergebnisse aus der makroskopischen Betrachtung für die Mikro-Simulation genutzt. Die Ausgangssituation wird in der Mikro-Simulation mit den notwendigen Randbedingungen und Parametern modelliert. Als Eingangsgrößen werden die Stellgrößen aus der Optimierung übergeben.

\subsection{Vorgehen zur Bewertung der Ergebnisse}

Der erste Schritt zur Bewertung der Optimierungsergebnisse ist die Analyse der Zielgröße, die anhand der MakroSimulation optimiert wurde. Wenn sich die Ergebnisse in der Mikro-Simulation von den aus der Makro-Simulation berechneten Zielgrößen unterscheiden, ist dies in Effekten begründet, die im Makro-Modell nicht betrachtet werden. In diesem Fall sollte die Optimierung mit neuen Einschränkungen wiederholt werden. Die Einschränkungen können entweder veränderte Randbedingungen für die Optimierung sein oder Anpassungen an der Modellierung.

Wenn die Ergebnisse übereinstimmen, so werden weitere Bewertungsparameter betrachtet, um die Qualität des Layouts zu bewerten. Dabei werden Werte untersucht, die aus der Makro-Simulation nicht hervorgehen bspw., dass Stückgüter herunterfallen oder beschädigt werden. Auch in diesem Fall sollte die Optimierung mit neuen Einschränkungen wiederholt werden. Möglicherweise müssen neue Stellgrößen berücksichtigt werden, um die Zielgrößen zu erreichen.

Als letzter Schritt können leichte Variationen der Stellgrößen betrachtet werden, um sicherzustellen, dass es sich tatsächlich um die Optima des Layouts handelt. Dies ist insbesondere dann der Fall, wenn es um konkrete Positionen der Stückgüter geht. In der Makro-Simulation und folglich auch in der Optimierung ist beispielsweise nicht direkt erkennbar, ob sich in einem Bereich ein Stückgut befindet oder zwei Stückgüter zur Hälfte.

\section{Anwendungsbeispiel}

Bandfördersysteme werden in verschiedenen Bereichen wie der Fertigungsindustrie, der Lebensmittelindustrie, Recyclingindustrie oder im Bergbau umfassend und weit verbreitet eingesetzt $[15,16,28]$. In der Regel sind sie das effizienteste und wirtschaftlichste Mittel zum Transport von Stück- und Schüttgütern zwischen den Arbeitsstationen einer Fließproduktion [5]. Die Arbeitsstationen innerhalb einer Anlage haben unterschiedliche Arbeitsräume und Bearbeitungszeiten. Die Zuführung und Verteilung der Güter 
Abb. 2 a Layout des Materialflusssystems, b Materialfluss am Abweiser
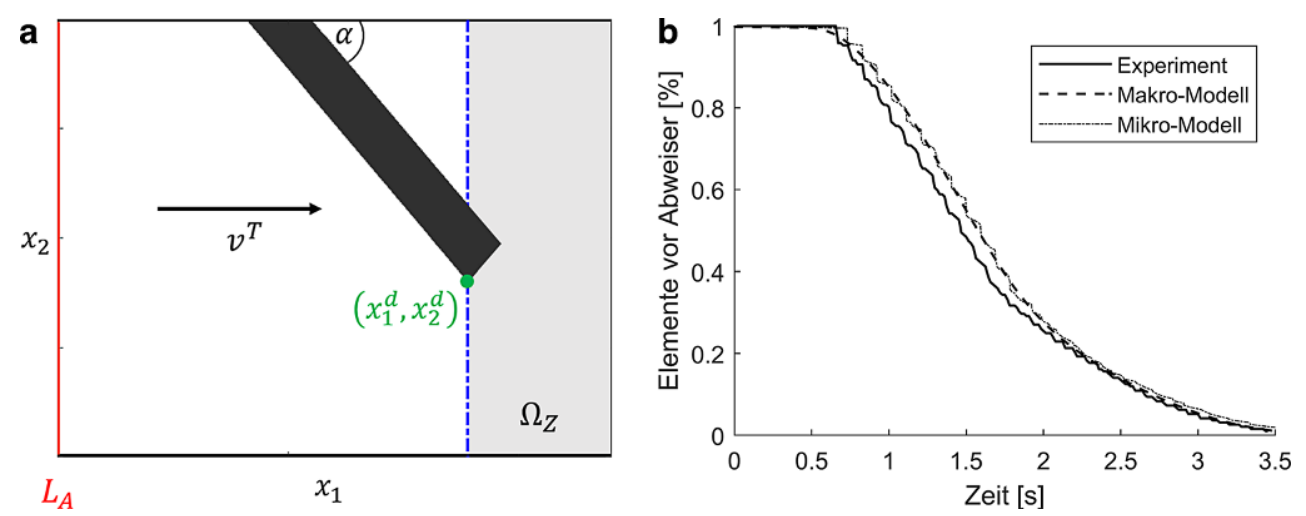

Abb. 3 Stückgutverteilung zum Zeitpunkt $\mathrm{T}=0,8 \mathrm{~s}$ in a MakroSimulation, b Mikro-Simulation b

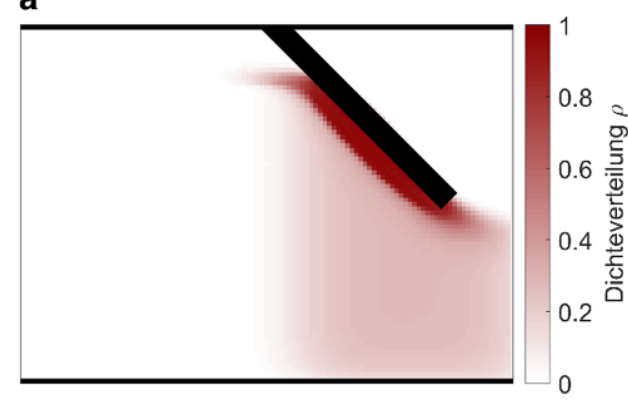

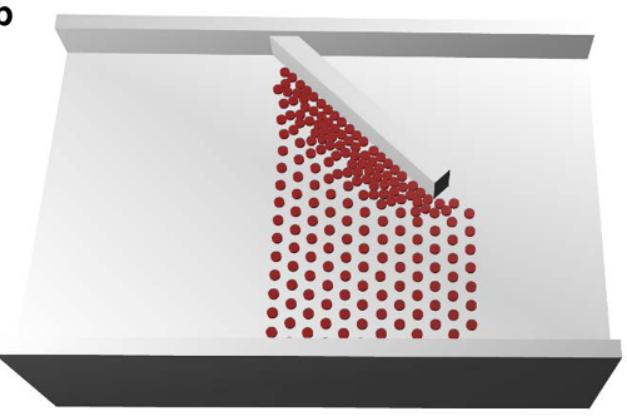

muss daher individuell erfolgen. Dies kann beispielsweise mit einer Kombination aus Bandförderern und Abweisern realisiert werden [13]. Die Abweiser dienen beispielsweise der Überführung von Stückgütern auf andere Bandförderer bzw. Transportsysteme innerhalb der Anlage. Im Folgenden wird zunächst die Steuerung des Materialflusses vor einer einzelnen Anlage betrachtet bevor im Abschn. 5.2 die Steuerung eines kombinierten Materialflusssystems betrachtet wird.

Es wird ein Materialflusssystem aus Bandförderern betrachtet, auf denen Stückgüter transportiert werden. Die Stückgüter werden an einem Abweiser abgelenkt, um sie zielgerichtet zu einer Bearbeitungsstation zu transportieren [7, 10]. Als Zielgröße wird eine Anzahl an Stückgütern hinter dem Abweiser zu einer vorgegebenen Zeit gesetzt. Die Stückgüter sollen dabei stets nebeneinander auf dem Bandförderer liegen und sich nicht überlagern. Es soll die Bandgeschwindigkeit als Stellgröße optimiert werden. Zunächst wird das Setting in der Makro-Simulation modelliert und das Optimierungsproblem gelöst, woraus sich eine optimale Bandgeschwindigkeit ergibt. Anschließend wird das Setting in der Mikro-Simulation modelliert und überprüft ${ }^{1}$. Dabei werden die makroskopisch optimalen Stellgrößen an die mikroskopische Simulation übergeben.

${ }_{1}$ Optimierung mit Matlab-Solver fmincon [14], Mikro-Simulation mit AgX Dynamics und Visual Studio 2015 als Compiler [1].

\subsection{Einfaches Materialflusssystem}

Es wird zunächst das Setting aus [7] zum Transport von $N=192$ zylindrischen Stückgütern mit Radius $\xi=0,012 \mathrm{~m}$ und Höhe $h=0,008 \mathrm{~m}$ in einer Zuführung bestehend aus einem Abweiser, um das Makro-Modell für unterschiedliche Bandgeschwindigkeiten zu validieren. Die Kombination aus Bandförderer und Abweiser ist in Abb. 2a schematisch dargestellt. Die Ansammlung der Stückgüter wird zunächst mit Geschwindigkeit $v^{T}$ auf dem Bandförderer $\left(x_{1}>L_{A}\right)$ transportiert bevor die Güter auf einen Abweiser treffen und kollidieren. Das Experiment ist abgeschlossen, wenn alle 192 Stückgüter den Abweiser passiert haben. Wie in Abb. 2b zu sehen und in den realen Messdaten von Göttlich et al. [7] ersichtlich, bilden die beiden Simulationsmodelle die realen Messdaten hinreichend gut ab (siehe [7] für weitere Vergleiche experimenteller Daten mit den Simulationsergebnissen). Im realen Experiment mit der Bandgeschwindigkeit $v^{T}=0,42 \mathrm{~m} / \mathrm{s}$ befinden sich zum Zeitpunkt $T=1 \mathrm{~s}$ alle Stückgüter vor dem Ende des Abweisers. Ziel ist nun, die Bandgeschwindigkeit so zu steuern, dass sich zum Zeitpunkt $T=1 \mathrm{~s}$ jeweils ein Viertel (Szenario S1), die Hälfte (S2) bzw. Dreiviertel (S3) der gesamten Stückgüter hinter dem Abweiser befinden $\left(\Omega_{z}=\left\{x \in \mathbb{R}^{2} \mid x_{1} \geq x_{1}^{d}\right\}\right)$. Die Bandgeschwindigkeit wird dabei für den gesamten Zeitraum konstant vorgegeben und für jedes Szenario das Optimierungsproblem (9)-(10) gelöst.

Nach der Optimierung in der Makro-Simulation (vgl. Abb. 3a) und der Verifikation in der Mikro-Simulation (vgl. 
Abb. 3b) ergeben sich die Werte in Tab. 1 jeweils für den Abweiserwinkel $\alpha_{1}=45 \mathrm{Grad}$ und $\alpha_{2}=60 \mathrm{Grad}$. Für beide Abweiserstellungen ergeben sich qualitativ gute Ergebnisse $^{2}$. Für die Szenarien S2 und S3 liegen die Ergebnisabweichungen (teilweise deutlich) unter $2 \%$. Für das Szenario S3 mit höheren Bandgeschwindigkeiten ist das Ergebnis zufriedenstellend, die Ergebnisabweichungen sind jedoch tendenziell höher. In der qualitativen Betrachtung der Ergebnisse anhand der Visualisierung und bei der Analyse der Bewegung in der dritten Dimension zeigt sich, dass die einzelnen Elemente wie gewünscht auf dem Bandförderer bleiben, sich nicht verklemmen und sich nicht überlagern. Die berechneten Bandgeschwindigkeiten erscheinen daher sinnvoll und das gewählte Layout passend. In allen Fällen kann nun eine Nachjustierung der Geschwindigkeiten im Mikro-Modell erfolgen, um den gewünschten Zielfunktionswert zu erreichen. Für S2 und die Abweiserstellung $\alpha_{2}$ ist eine weitere Anpassung obsolet, da hier die optimale Geschwindigkeit den exakten Zielfunktionswert im

\footnotetext{
2 Die makroskopisch bestimmte optimale Geschwindigkeit wird hier im mikroskopischen Modell validiert. In der Realität sollte jedoch berücksichtigt werden, in welchem Bereich die Bandgeschwindigkeit regelbar ist. Diese ist abhängig von der Steuerung und dem Motor. Der im Experiment [7] verwendete Motor kann als Anregelzeit $1 \mathrm{~ms}$ errreichen. Möglicherweise liegt die optimale Stellgröße des makroskopischen Modells bei anderen Varianten zwischen zwei diskreten Einstellungsmöglichkeiten des Bandförderers. Wenn die Einstellungsmöglichkeiten bekannt sind, sollten im mikroskopischen Modell nur innerhalb der zulässigen Einstellungen überprüft werden, welche Einstellung mikroskopisch den besten Zielfunktionswert liefert.
}

Mikro-Modell erreicht. Die Rechenzeit der beiden Simulationsmodelle für die Simulation über den gesamten Zeithorizont $\mathrm{T}=3,5 \mathrm{~s}$ des Anwendungsbeispiel wird auf einem PC mit Intel ${ }^{\circledR}$ Core ${ }^{\mathrm{TM}}$ i7-7700HQ mit 2,8 GHz Prozessor (vier Kerne, acht logische Prozessoren, 8,0GB RAM) verglichen. Gemessen wird die Rechenzeit ohne Initialisierung, Visualisierung und Datenspeicherung. Für das Mikro-Modell $(\Delta t=0,001 \mathrm{~s})$ liegt die Rechenzeit bei durchschnittlich $114,2 \mathrm{~s}$. Für das Makro-Modell $(\Delta t=0,001 \mathrm{~s}, \Delta x=0,01 \mathrm{~m})$ beträgt die durchschnittliche Rechenzeit 24,5s. Die Vorteilhaftigkeit des Makro-Modells zeigt sich sowohl in der Berechnungszeit über einen kompletten Simulationslauf als auch in der benötigten Zeit zur Berechnung einzelner Zeitschritte. Während die Berechnungszeit im mikroskopischen Modell abhängig von der Anzahl der Kollisionen ist und stark variieren kann, ist die Berechnungszeit je Zeitschritt im Makro-Modell konstant und deterministisch. Somit ergibt sich insbesondere ein Vorteil in der Berechnung von Stauzonen mit vielen Kollisionen. Da das makroskopische Modell zur Optimierung der Bandgeschwindigkeit genutzt wird, in der die Simulationsroutine mehrfach aufgerufen wird, ergibt sich insgesamt ein deutlicher Zeitvorteil.

\subsection{Kombiniertes Materialflusssystem}

Für die Optimierung des Materialflusses einer Anlage stellt die Betrachtung einzelner Stationen nur einen kleinen Ausschnitt dar. Zusätzlich muss die Kombination mehrerer Stationen hintereinander untersucht werden. Diese Stationen bearbeiten häufig Chargen unterschiedlicher Größe in un-

Tab. 1 Ergebnisse aus Optimierung und Validierung

\begin{tabular}{|c|c|c|c|c|c|}
\hline \multirow[b]{2}{*}{ Szenario } & \multirow[b]{2}{*}{$\begin{array}{l}\text { Zielgröße [Stückgü- } \\
\text { ter nach Abweiser] }\end{array}$} & \multicolumn{2}{|c|}{ Winkel des Abweisers $\alpha_{1}=45^{\circ}$} & \multicolumn{2}{|c|}{ Winkel des Abweisers $\alpha_{2}=60^{\circ}$} \\
\hline & & $\begin{array}{l}\text { Stellgröße [Bandge- } \\
\text { schwindigkeit in } \mathrm{m} / \mathrm{s} \text { ] }\end{array}$ & $\begin{array}{l}\text { Mikro-Simulation [Ele- } \\
\text { mente nach Abweiser] }\end{array}$ & $\begin{array}{l}\text { Stellgröße [Bandge- } \\
\text { schwindigkeit in } \mathrm{m} / \mathrm{s} \text { ] }\end{array}$ & $\begin{array}{l}\text { Mikro-Simulation [Ele- } \\
\text { mente nach Abweiser] }\end{array}$ \\
\hline S1 & $144(75 \%)$ & 0,860 & $137(71,35 \%)$ & 1,109 & $132(68,75 \%)$ \\
\hline $\mathrm{S} 2$ & $96(50 \%)$ & 0,651 & $93(48,44 \%)$ & 0,686 & $96(50 \%)$ \\
\hline S3 & $48(25 \%)$ & 0,488 & $47(24,88 \%)$ & 0,461 & $51(26,56 \%)$ \\
\hline
\end{tabular}

Abb. 4 Anwendungsfall der Bewegung von Flaschen auf einem Bandförderer in einer Abfüllanlage

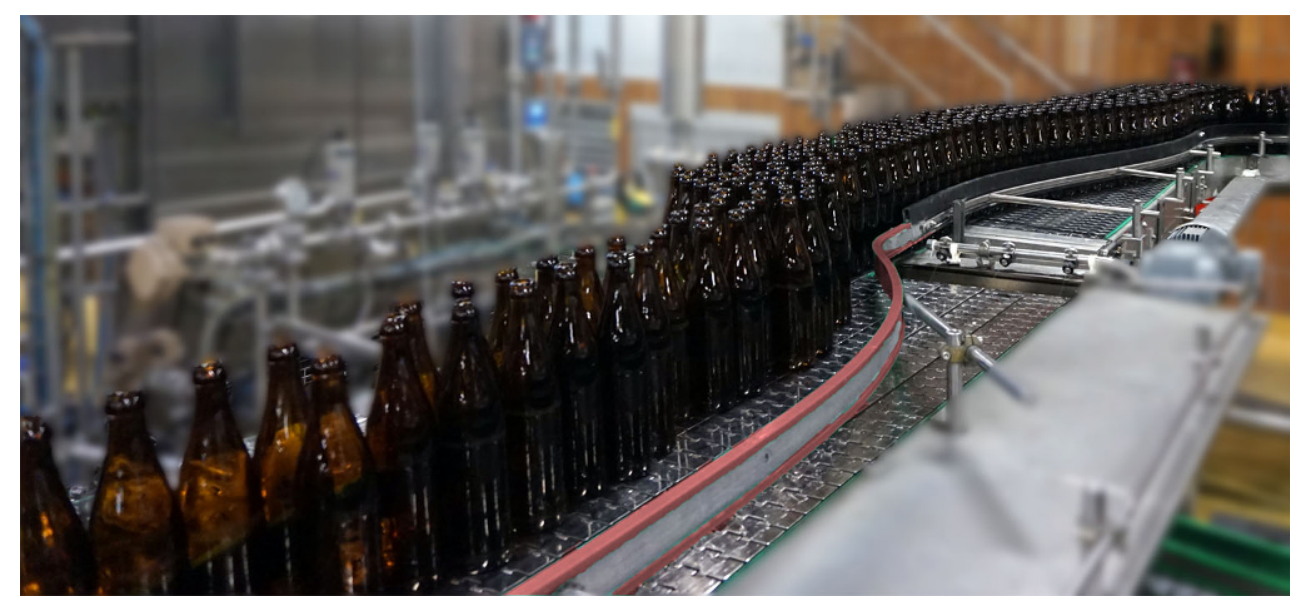


terschiedlicher Zeit und die Bearbeitung an vorherigen Stationen beeinflusst die Materialversorgung der nachfolgenden Arbeitsstationen. Jede einzelne Station benötigt möglichst kontinuierlichen Nachschub, damit keine Leerlaufzeiten entstehen [3]. Eine exemplarische Anwendung sind Getränkeabfüllanlagen zur Befüllung von Flaschen (siehe Abb. 4 und Beschreibung von Sorgatz [24]). Die Flaschen bewegen sich in einer Ebene und werden mithilfe eines komplexen Netzwerkes aus Bandförderern zwischen verschiedenen Stationen transportiert. Die Stationen umfassen bspw. die Reinigung, Inspektion, Befüllung, Verschluss, Etikettierung und Kontrolle der Flaschen [26]. Da diese Stationen in der Regel unterschiedliche Kapazitäten und Bearbeitungszeiten haben, werden die Flaschen auf den Bandförderern entsprechend bewegt, um eine dosierte Befüllung nachgelagerter Flächen zu erreichen [13]. Dies wird unter anderem mit Abweisern oder Bandverengungen realisiert.

Im Folgenden wird eine Erweiterung des Szenarios aus dem vorherigen Abschnitt betrachtet. Anstelle einer einzelnen Zuführung wird eine Hintereinanderschaltung mehrerer Stationen $m=1, \ldots M, M>1$ innerhalb eines Fertigungssystems untersucht. Dabei wird angenommen, dass eine $\mathrm{Zu}-$ führung die Stückgüter mit einem Bandförderer zur nächsten Arbeitsstation transportiert, in die die Stückgüter mit einem Abweiser gelenkt werden, wie in Abb. 5 schematisch für $M=2$ gezeigt. Die Größen des Beispielszenarios sind in Tab. 2 zusammengefasst. Dabei ist die Bandgeschwindigkeit des Bandförderers $v_{m, 1}=v_{m, 1}^{T}, m=1,2$ regelbar (im Folgenden wird zur Übersichtlichkeit auf den oberen Index $T$ verzichtet). Das Zielgebiet einer Arbeitsstation $m$, gegeben durch $\Omega_{Z_{m}}$, ist in Abb. 5 grau hinterlegt. Relevant für den Materialfluss ist, dass die Gebiete ebenfalls Bandförderer mit Geschwindigkeit sind. Jede Arbeitsstation hat gegebene Bedarfe $b_{m}$ zu vorgegebenen Zeitpunkten $t_{m}^{*}$. Nach Bearbeitung der Güter in der Station $m$ werden die Güter zur nächsten Station $m+1$ transportiert. Diese weist möglicherweise andere Bedarfe zu anderen Zeitpunkten auf. Auch im Folgenden ist das Szenario der Übersichtlichkeit wegen auf $M=2$ Arbeitsstationen beschränkt. Es wird angenommen, dass Station 1 jeweils Chargen in der Größe von $C_{1}=800$ Stückgüter simultan bearbeiten kann, während Station 2 nur Chargen von $C_{2}=200$ Stückgütern bearbeiten kann, jedoch eine kürzere Bearbeitungszeit hat. Während der Bearbeitung steht das Förderband in der Station still. Nach der Bearbeitung werden die Stationen geleert, bevor neue Stückgüter hinein transportiert werden können. Es wird ein vereinfachtes Szenario betrachtet, bei dem die Auswirkungen des Stoppens und Anfahrens der Bänder vernachlässigt werden.

Auf dem Bandförderer vor Station 1 befinden sich zur Startzeit $t=0$ insgesamt 1000 Stückgüter. Die restlichen Bandförderer sind leer. Es wird zunächst die Optimierung der Bandgeschwindigkeit $v_{1,1}^{*}=v_{1,2}^{*}$ betrachtet, so dass die Station 1 zum Zeitpunkt $t_{1}^{*}=5 \mathrm{~s}$ mit der Bearbeitung beginnen kann. Zu Beginn wird vorausgesetzt, dass alle Bandförderer die selbe Geschwindigkeit haben $v_{m, 1}=v_{m, 2}=$ $v_{1,1}^{*}, m=1,2$. Anschließend werden die Güter auf das Band vor der Station 2 transportiert. Die Bearbeitung in der Station ist abgeschlossen, wenn die Station komplett entleert ist. Sobald alle Güter die Station verlassen haben, beginnt die Optimierung der Bandgeschwindigkeit, sodass 2 s später die erste Charge mit $C_{2}=200$ Stückgütern in der Station 2 angekommen sein soll. Die nächste Charge wiederum soll $2 \mathrm{~s}$ nach der Bearbeitung der ersten Charge in der Station sein. Die Bandgeschwindigkeiten für den Transport der Chargen sollen hierbei optimal bestimmt werden. Für jede Charge wird ein Optimierungsproblem der Form (9)-(10) gelöst.

Das Vorgehen lässt sich folgendermaßen zusammenfassen: Die makroskopische Optimierung erhält zunächst die initiale Positionierung der Güter als Eingangsgröße. Da-

Abb. 5 Layout des kombinierten Materialflusssystems

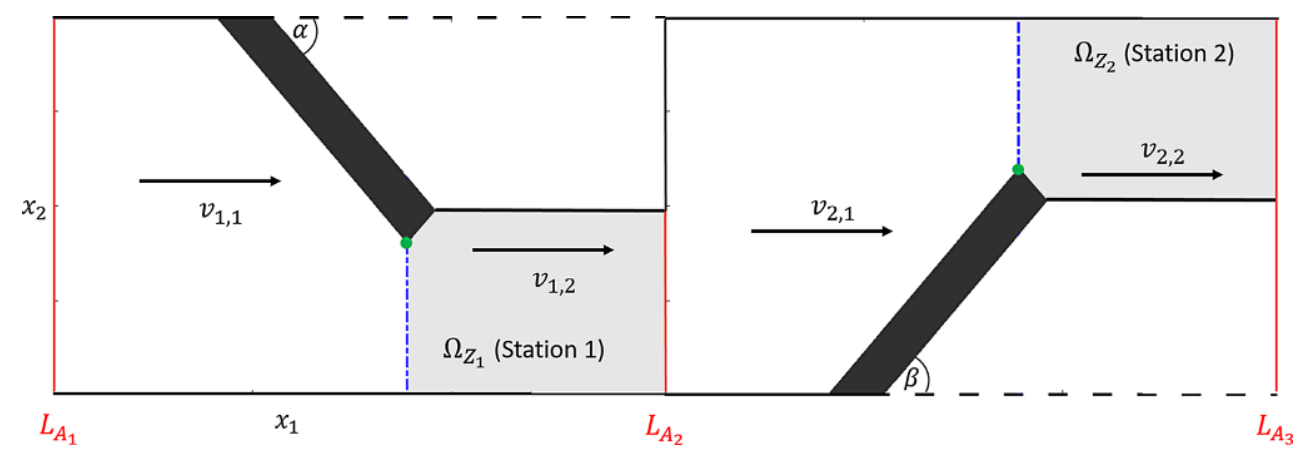

Tab. 2 Größenangaben des kombinierten Materialflusssystems

\begin{tabular}{lllll}
\hline & Band 1 & Station 1 & Band 2 & Station 2 \\
\hline Länge & $3 \mathrm{~m}$ & $3 \mathrm{~m}$ & $3 \mathrm{~m}$ & $3 \mathrm{~m}$ \\
Breite & $0,8 \mathrm{~m}$ & $0,4 \mathrm{~m}$ & $0,8 \mathrm{~m}$ & $0,4 \mathrm{~m}$ \\
Winkel des Abweisers & $\alpha=45 \mathrm{Grad}$ & - & $\beta=45 \mathrm{Grad}$ & - \\
\hline
\end{tabular}


rauf aufbauend liefert diese die makroskopisch optimale Geschwindigkeit $v^{*}$. Anschließend wird dieses Ergebnis in der mikroskopischen Simulation überprüft, welche die makroskopisch optimale Geschwindigkeit $v^{*}$ als Eingabe erhält. Falls möglich wird die Geschwindigkeit auf den Wert $v^{* *}$ angepasst, welche mikroskopisch den besten Zielfunktionswert liefert. Die Positionen der Stückgüter, die sich durch die mikroskopische Simulation ergeben, werden dem makroskopischen Modell anschließend als initiale Positionierung für die nächste Optimierung übergeben. Dadurch ergibt sich in diesem Szenario eine Kopplung der einzelnen Optimierungen durch die Startposition der Güter. Das Umschalten zwischen den Simulationsebenen erfolgt (Stand heute) manuell.

Die Optimierungsergebnisse $v^{*}$ und die nachjustierten Geschwindigkeiten $v^{* *}$ sind in Tab. 3 für die einzelnen Chargen dargestellt. Nach der ersten Optimierung, die die makroskopische Geschwindigkeit von $v_{1,1}^{*}=0,576 \mathrm{~m} / \mathrm{s}$ ergibt, liefert die Auswertung der mikroskopischen Simulation 820 Stückgüter in Station 1 zum Zeitpunkt $t_{1}^{*}=5 \mathrm{~s}$. Durch Nachjustierung der Geschwindigkeit kann das Ergebnis auf 799 Stückgüter verbessert werden. Die optimale Anzahl von $C_{1}=800$ Stückgütern wird in der MikroModellierung mit Nachjustierung bei der Geschwindigkeit im Bereich der Tausendstel nicht erreicht. Nach der ersten Optimierung zum Zeitpunkt $t=5 \mathrm{~s}$ befinden sich vor der Station 1 nur noch 201 Stückgüter, deren Anzahl nicht ausreichend für eine erneute Bearbeitung der Station 1 ist. Nachdem der Transport der ersten Charge zu Band 3 abgeschlossen ist, wird daher keine erneute Optimierung der Geschwindigkeiten für Station 1 betrachtet. Nach dem Transport der Güter zur nächsten Zuführung erfolgt eine erneute Optimierung, um die Bandgeschwindigkeit für die gewünschte Anzahl von 200 Stückgütern in der Station 2 zu bestimmen. Die Anfangspositionierung der Güter ergibt sich hierbei aus dem Resultat der mikroskopischen Simula- tion zu dem Zeitpunkt, an dem alle Güter auf der Zuführung angekommen sind. Die Ergebnisse für die erste Charge an Station 1 und die ersten drei Chargen an Station 2 sind in Tab. 3 zusammengefasst.

Insgesamt beträgt die Abweichung der Ergebnisse des makroskopischen Modells maximal 20 Stückgüter und kann durch Nachjustierung auf ein Stückgut verbessert werden. Die Nachjustierung der Ergebnisse ist aufwendig dadurch, dass jede minimale Änderung separat getestet werden muss. Darüber hinaus ist das Verhältnis zwischen Bandgeschwindigkeit und passierten Stückgütern nicht proportional. Je nach Anstauung der Stückgüter können höhere Geschwindigkeiten zu geringeren Anzahlen an Stückgütern zu bestimmten Zeitpunkten in den Stationen führen.

Variationen des obigen Szenarios als Erweiterungen sind ebenfalls möglich. Anstelle des einfachen Transports durch eine Station hindurch, könnten die Stückgüter während der Bearbeitung vom Band genommen werden und danach auf den Zuführer in der zweiten Station gesetzt werden. In diesem Falle könnte bei Kenntnis der Positionen, auf die die Güter nach Bearbeitung gesetzt werden, die zweiten Optimierung getrennt von der ersten durchgeführt werden.

\section{Fazit}

Das Anwendungsbeispiel zeigt, dass die Optimierung für die Anwendungsfälle zufriedenstellende Ergebnisse liefert. Die optimale Steuerung ist im Bereich höherer Stückzahlen nicht offensichtlich und eine manuelle Auswahl von Stellgrößen wird bereits bei kleinen Anlagenbeispielen komplex. Durch die mathematische Optimierung anhand des makroskopischen Modells können die Stellgrößen ohne Vorkenntnisse bestimmt werden. Sie erlaubt die Bestimmung einer Stellgröße im kontinuierlichen Lösungsraum mit verhältnismäßig geringer Abweichung von der optima-

Tab. 3 Ergebnisse des kombinierten Anwendungsbeispiels

\begin{tabular}{|c|c|c|c|c|c|c|c|c|c|}
\hline \multirow[t]{3}{*}{ Phase } & \multirow[t]{3}{*}{ Zeitdauer [s] } & \multicolumn{2}{|l|}{ Band 1} & \multicolumn{2}{|c|}{ Station 1} & \multicolumn{2}{|l|}{ Band 2} & \multicolumn{2}{|c|}{ Station 2} \\
\hline & & Makro & Mikro & Makro & Mikro & Makro & Mikro & Makro & Mikro \\
\hline & & $v_{1,1}^{*}$ & $v_{1,1}^{* *}$ & $v_{1,2}^{*}$ & $v_{1,2}^{* *}$ & $v_{2,1}^{*}$ & $v_{2,1}^{* *}$ & $v_{2,2}^{*}$ & $v_{2,2}^{* *}$ \\
\hline Charge 1 zu Station 1 & 5 & 0,576 & 0,562 & $=v_{1,1}^{* *}$ & & & & & \\
\hline Ziel 1 & \multicolumn{9}{|c|}{ Stückgüter in Station 1: Zielgröße 800, Realisiert $820^{*}$ bzw. $799 * *$} \\
\hline Charge $1 \mathrm{zu}$ Band 2 & n.r. & $=0$ & & $=v_{1.1}^{* *}$ & & & & & \\
\hline Charge 1 zu Station 2 & 2 & - & n.r. & - & n.r. & 0,900 & 0,922 & - & $=v_{2,1}^{* *}$ \\
\hline Ziel 2 & \multicolumn{9}{|c|}{ Stückgüter in Station 2: Zielgröße 200, Realisiert 192* bzw. 199** } \\
\hline Transport zu Band 5 & n.r. & - & n.r. & - & n.r. & - & 0 & - & $=v_{2,1}^{* *}$ \\
\hline Charge 2 zu Station 2 & 2 & - & n.r. & - & n.r. & 0,268 & 0,262 & - & $=v_{2,1}^{* *}$ \\
\hline Ziel 3 & \multicolumn{9}{|c|}{ Stückgüter in Station 2: Zielgröße 200, Realisiert 203* bzw. 201** } \\
\hline Transport zu Band 5 & n.r. & - & n.r. & - & n.r. & - & 0 & - & $=v_{2,1}^{* *}$ \\
\hline Charge 3 zu Station 2 & 2 & - & n.r. & - & n.r. & 0,265 & 0,295 & - & $=v_{2,1}^{* *}$ \\
\hline Ziel 4 & \multicolumn{9}{|c|}{ Stückgüter in Station 2: Zielgröße 200, Realisiert $180^{*}$ bzw. 199** } \\
\hline
\end{tabular}

n. $r$. nicht relevant. 
len Zielgröße in kurzer Zeit. Die mathematische Optimierung lässt sich zudem durch die Verwendung des makroskopischen Simulationsmodells ohne erhöhten Aufwand auf höhere Stückgutzahlen anwenden. Hier kann der Vorteil des Makro-Modells ausgenutzt werden, da der Rechenaufwand konstant ist während die Simulationszeiten des Mikro-Modells stark zunehmen [7]. Die Kombination der beiden Simulationsarten verbessert die reine Optimierung über das makroskopischen Modell. Durch die Hinzunahme der mikroskopischen Simulation werden Abweichungen von maximal $10 \%$ auf maximal $0,5 \%$ Abweichung vom Zielwert verbessert. Es kann davon ausgegangen werden, dass die mikroskopischen Simulationsergebnisse dem realen Materialfluss in einer Anlage entsprechen und die geringe Abweichung mit den Vorgaben folglich auch in Realität erreicht wird. Auf diese Weise sind sowohl die Berechnungen für die Optimierungen möglich, als auch die Berücksichtigung physikalischer Effekte, die im Makro-Modell nicht erkannt werden. Die Kombination der makroskopischen Optimierung und Validierung durch das mikroskopische Modell ist vor allem in Szenarien relevant, bei denen die Leistung einer Anlage durch die Variation von Steuergrößen verbessert werden kann. Die Optimierung der Steuerung über das makroskopische Modell erlaubt eine Menge an verschiedenen Förderbandkombinationen (bspw. Breiten, Längen, Abweiserwinkel ...) und Szenarien mit unterschiedlicher Stückgutanzahl effizient zu optimieren. Die ergänzende Verwendung des mikroskopischen Modells ermöglicht die Validierung der optimalen Ergebnisse für die Vielzahl an Szenarien, in denen keine Realdaten vorhanden sind.

\section{Zusammenfassung und Ausblick}

In dieser Arbeit wurde ein Konzept entwickelt, um ein Materialflusssystem bestehend aus Bandförderern und Abweisern mathematisch für eine optimale Bewegung der Stückgüter zu optimieren. Dabei werden Stellgrößen anhand einer makroskopischen Simulation auf einen Zielwert optimiert und anhand einer mikroskopischen Simulation validiert und bei Bedarf verbessert. Die makroskopische Simulation basiert auf einer partielle Differentialgleichung in Erhaltungsform, die Materialfluss in zwei Dimensionen beschreibt. Sie ermöglicht schnelle Optimierungen der Bandgeschwindigkeit, die bisher nicht umsetzbar waren. Die mikroskopische Simulation ist eine physikbasierte Starrkörpersimulation im dreidimensionalen Raum, durch die zusätzliche Effekte Berücksichtigung finden.

Aus der Nutzung der zwei Modellarten in dem vorgestellten Konzept ergibt sich ein großer Modellierungsaufwand, da jedes Szenario doppelt (makroskopisch und mikroskopisch) modelliert werden muss. Inhalt weiterer For- schung ist daher eine automatische, bidirektionale Überführung beider Modellarten zur Reduzierung des Modellierungsaufwands. Eine allgemeine Klassifikation, für welche Anwendungsfälle eine Mikro-Simulation zur Verifikation und Verbesserung der Ergebnisse notwendig bzw. hilfreich ist, wurde bisher nicht untersucht. In einigen Fällen kann vermutlich auf diese Simulationsart verzichtet werden, während in anderen Fällen die Makro-Simulation unzureichende Ergebnisse liefert. In der vorliegenden Betrachtung erwiesen sich die Ergebnisse des Makro-Modells für die betrachteten zylindrische Güter als hinreichend. Für die Bewegung eckiger Stückgüter wurde das Makro-Modell ebenfalls validiert und als geeignet befunden [19], sodass Optimierungen auch für eckige Stückgüter vorgenommen werden könnten. Speziell die Modellierung des Abweisers als Rand des Gebietes, was eine Modellierung des Abweisers unabhängig von der Bandgeschwindigkeit ermöglichen soll, wird separat in einer zukünftigen Publikation untersucht.

Kompliziertere Layouts, die mathematisch herausfordernder sind bspw. mit einer größeren Anzahl an Bandförderern mit Pufferzonen und unterschiedlichen Geschwindigkeiten der Bandförderer, können in Folge betrachtet werden. Zusätzliche Stellgrößen sowie Nebenbedingungen können eingeführt werden. Anstelle der Optimierung der Bandgeschwindigkeit kann zusätzlich auch eine optimale initiale Positionierung der Güter auf einzelnen Zuführungen mit dem makroskopischen Modell berechnet werden (siehe [4]). Zukünftig wird eine Hardware-in-the-Loop Simulation mit einer realen Steuerung betrachtet, um die echtzeitkritische Berechnung des Simulationsmodells zu untersuchen. Aufbauend darauf könnte das vorhandene Konzept genutzt werden, um anhand von Echtzeitdaten über den Materialfluss, die Bandgeschwindigkeit von Bandförderern in Echtzeit anzupassen, um eine geplante Taktung/Auslastung von Materialflusssystemen zu erreichen.

Förderung Gefördert durch die Deutsche Forschungsgemeinschaft (DFG) - Projektnummer 327964174.

Funding Open Access funding enabled and organized by Projekt DEAL.

Interessenkonflikt A. Kienzlen, J. Weißen, A. Verl und S. Göttlich geben an, dass kein Interessenkonflikt besteht.

Open Access Dieser Artikel wird unter der Creative Commons Namensnennung 4.0 International Lizenz veröffentlicht, welche die Nutzung, Vervielfältigung, Bearbeitung, Verbreitung und Wiedergabe in jeglichem Medium und Format erlaubt, sofern Sie den/die ursprünglichen Autor(en) und die Quelle ordnungsgemäß nennen, einen Link zur Creative Commons Lizenz beifügen und angeben, ob Änderungen vorgenommen wurden.

Die in diesem Artikel enthaltenen Bilder und sonstiges Drittmaterial unterliegen ebenfalls der genannten Creative Commons Lizenz, sofern sich aus der Abbildungslegende nichts anderes ergibt. Sofern das betreffende Material nicht unter der genannten Creative Commons Lizenz steht und die betreffende Handlung nicht nach gesetzlichen Vorschrif- 
ten erlaubt ist, ist für die oben aufgeführten Weiterverwendungen des Materials die Einwilligung des jeweiligen Rechteinhabers einzuholen.

Weitere Details zur Lizenz entnehmen Sie bitte der Lizenzinformation auf http://creativecommons.org/licenses/by/4.0/deed.de.

\section{Literatur}

1. AGX Dynamics 2.22.1.0. Algoryx Simulation AB, Umeå, Sweden

2. Betts JT, Campbell SL (2005) Discretize then optimize. In: Ferguson DR, Peters TJ (Hrsg) Mathematics for industry: challenges and frontiers. SIAM Society for Industrial and Applied Mathematics, Philadelphia, S 140-157

3. Braglia M (2001) Designing a belt conveyor controller in a bottling plant using fuzzy logic and genetic algorithms. Packag Technol Sci 14(6):231-248. https://doi.org/10.1002/pts.554

4. Erbrich M, Göttlich S, Pfirsching M (2018) Optimal packing of material flow on conveyor belts. Optim Eng 19(1):71-96. https://doi. org/10.1007/s11081-017-9362-5

5. Festa A, Göttlich S, Pfirsching M (2019) A model for a network of conveyor belts with discontinuous speed and capacity. Networks Heterog Media 14(2):389-410

6. Göttlich S, Pfirsching M (2018) A micro-macro hybrid model with application for material and pedestrian flow. Cogent Math Stat. https://doi.org/10.1080/25742558.2018.1476049

7. Göttlich S, Hoher S, Schindler P, Schleper V, Verl A (2014) Modeling, simulation and validation of material flow on conveyor belts. Appl Math Model 38(13):3295-3313. https://doi.org/10.1016/j. apm.2013.11.039

8. Göttlich S, Klar A, Tiwari S (2015) Complex material flow problems. A multi-scale model hierarchy and particle methods. J Eng Math 1:15-29. https://doi.org/10.1007/s10665-014-9767-5

9. Günthner W, Kadachi M (2001) Simulationsgestützte Planung und Nutzung von Getränke-Abfüllanlagen. Abschlussbericht, Technische Universität München

10. Hoher S, Schindler P, Göttlich S, Schleper V, Röck S (2012) System dynamic models and real-time simulation of complex material flow systems. In: ElMaraghy HA (Hrsg) Enabling manufacturing competitiveness and economic sustainability. Springer, Berlin, Heidelberg, S 316-321

11. Krug W, Rose O (2011) Optimierung. In: März L, Krug W, Rose O, Weigert G (Hrsg) Simulation und Optimierung in Produktion und Logistik. Springer Berlin Heidelberg, Berlin, Heidelberg, S 21-28

12. Lee CG, Park SC (2014) Survey on the virtual commissioning of manufacturing systems. J Comput Des Eng 1(3):213-222. https:// doi.org/10.7315/JCDE.2014.021

13. Martin H (2011) Stetigförderer. In: Martin H (Hrsg) Transport- und Lagerlogistik. Vieweg+Teubner Verlag, Wiesbaden, S 132-213

14. MATLAB and Optimization Toolbox Release (2019) The MathWorks, Inc., Natick, Massachusetts, United States
15. Mattone R, Campagiorni G, Galati F (2000) Sorting of items on a moving conveyor belt. Part 1: a technique for detecting and classifying objects. Robot Comput Integr Manuf 16(2-3):73-80. https:// doi.org/10.1016/S0736-5845(99)00040-X

16. McNearny RL, Nie Z (2000) Simulation of a conveyor belt network at an underground coal mine. Min Res Eng 09(03):343-355. https:// doi.org/10.1142/S0950609800000299

17. Naik SB, Kallurkar S (2016) A literature review on efficient plant layout design. Int J Ind Eng 7(2):43-51

18. Pourhassan MR, Raissi S (2017) An integrated simulation-based optimization technique for multi-objective dynamic facility layout problem. J Ind Inf Integr 8:49-58. https://doi.org/10.1016/j.jii. 2017.06.001

19. Prims D, Kötz J, Göttlich S, Katterfeld A (2019) Validation of flow models as new simulation approach for parcel handling in bulk mode. Logistics. Journal. https://doi.org/10.2195/lj_Rev_prims_ en_201906_01

20. Puntel Schmidt P (2017) Methoden zur simulationsbasierten Absicherung von Steuerungscode fertigungstechnischer Anlagen. Dissertation, Helmut-Schmidt-Universität

21. Rolle RP, Martucci VO, Godoy EP (2019) Digitalization of manufacturing processes: proposal and experimental results workshop on metrology for Industry 4.0 and IoT (metroInd4.0\&IoT). IEEE, S 426-431. https://doi.org/10.1109/METROI4.2019.8792838

22. Rossi E, Weissen J, Goatin P, Göttlich S (2019) Well-posedness of a non-local model for material flow on conveyor belts. ESAIM: M2AN https://doi.org/10.1051/m2an/2019062

23. Scheifele C, Lechler A, Verl A (2016) Materialflussmodelle für die HiL-Simulation. Wt Werkstattstech Online 106(3):119-124

24. Sorgatz A (2013) Stufenlose Regelung von Materialflusssystemen am Beispiel von Behältertransporteuren in Getränkeabfüllanlagen. Dissertation, Technische Universität München

25. VDI Verein Deutscher Ingenieure (2016) Virtuelle Inbetriebnahme - Modellarten und Glossar 25.040.40, 35.240.50(3693 - Blatt 1). Beuth, Berlin

26. Voigt T (2004) Neue Methoden für den Einsatz der Informationstechnologie bei Getränkeabfüllanlagen. Dissertation, Technische Universität München

27. Zäh MF, Wünsch G, Hensel T, Lindworsky A (2006) Nutzen der virtuellen Inbetriebnahme: Ein Experiment. ZWF 101(10):595-599. https://doi.org/10.3139/104.101070

28. Zeng F, Wu Q (2015) A prediction model for the energy consumption of a Belt Conveyor System Based on Neural Network IEEE International Conference on Information and Automation (ICIA). IEEE, Piscataway, NJ, S 1865-1870

29. Zhang Z, Wang X, Wang X, Cui F, Cheng H (2019) A simulationbased approach for plant layout design and production planning. J Ambient Intell Human Comput 10(3):1217-1230. https://doi.org/ 10.1007/s12652-018-0687-5 